\title{
Novel cancer-testis antigen expression on glioma cell lines derived from high-grade glioma patients
}

\author{
YASUTO AKIYAMA $^{1 *}$, MASARU KOMIYAMA ${ }^{1 *}$, HARUO MIYATA $^{1}$, MIKA YAGOTO ${ }^{1}$, TADASHI ASHIZAWA ${ }^{1}$, \\ AKIRA IIZUKA $^{1}$, CHIE OSHITA ${ }^{1}$, AKIKO KUME ${ }^{1}$, MASAHIRO NOGAMI ${ }^{1}$, ICHIRO ITO ${ }^{2}$, REIKO WATANABE ${ }^{2}$, \\ TAKASHI SUGINO ${ }^{2}$, KOICHI MITSUYA ${ }^{3}$, NAKAMASA HAYASHI ${ }^{3}$, YOKO NAKASU ${ }^{3}$ and KEN YAMAGUCHI ${ }^{1}$ \\ ${ }^{1}$ Immunotherapy Division, Shizuoka Cancer Center Research Institute, ${ }^{2}$ Division of Pathology, \\ ${ }^{3}$ Division of Neurosurgery, Shizuoka Cancer Center Hospital, Sunto-gun, Shizuoka 411-8777, Japan
}

Received December 10, 2013; Accepted January 29, 2014

DOI: $10.3892 /$ or.2014.3049

\begin{abstract}
Glioblastoma multiforme (GBM) is one of the most malignant and aggressive tumors, and has a very poor prognosis with a mean survival time of $<2$ years, despite intensive treatment using chemo-radiation. Therefore, novel therapeutic approaches including immunotherapy have been developed against GBM. For the purpose of identifying novel target antigens contributing to GBM treatment, we developed 17 primary glioma cell lines derived from high-grade glioma patients, and analyzed the expression of various tumor antigens and glioma-associated markers using a quantitative PCR and immunohistochemistry (IHC). A quantitative PCR using 54 cancer-testis (CT) antigen-specific primers showed that $36 \mathrm{CT}$ antigens were positive in at least 1 of 17 serumderived cell lines, and 17 antigens were positive in $>50 \%$ cell lines. Impressively, 6 genes (BAGE, MAGE-A12, CASC5, CTAGE1, DDX43 and IL-13RA2) were detected in all cell lines. The expression of other 13 glioma-associated antigens than CT genes were also investigated, and 10 genes were detected in $>70 \%$ cell lines. The expression of CT antigen and glioma-associated antigen genes with a high frequency were also verified in IHC analysis. Moreover, a relationship of antigen gene expressions with a high frequency to overall survival was investigated using the Repository of Molecular Brain Neoplasia Data (REMBRANDT) database of the
\end{abstract}

Correspondence to: Dr Yasuto Akiyama, Immunotherapy Division, Shizuoka Cancer Center Research Institute, 1007 Shimonagakubo, Nagaizumi-cho, Sunto-gun, Shizuoka 411-8777, Japan

E-mail: y.akiyama@scchr.jp

"Contributed equally

Abbreviations: GBM, glioblastoma multiforme; TMZ, temozolomide; MGMT, $\mathrm{O}^{6}$-methylguanine-DNA-methyltransferase; IHC, immunohistochemistry; RT-PCR, reverse transcriptase-polymerase chain reaction; $\mathrm{CT}$ antigen, cancer-testis antigen

Key words: cancer-testis antigen, high-grade glioma, quantitative PCR, immunohistochemistry, glioma-related gene expression database
National Cancer Institute, and expression of 6 genes including IL-13RA2 was inversely correlated to overall survival time. Furthermore, 4 genes including DDX43, TDRD1, HER2 and gp100 were identified as MGMT-relevant factors. In the present study, several CT antigen including novel genes were detected in high-grade glioma primary cell lines, which might contribute to developing novel immunotherapy and gliomaspecific biomarkers in future.

\section{Introduction}

Glioblastoma multiforme (GBM) is one of the most malignant and aggressive tumors, and has a very poor prognosis and high recurrence rate with a mean survival time of less than 2 years even after the recent development of an intensive temozolomide (TMZ)-based treatment protocol $(1,2)$. Thus, a novel therapeutic approach to controlling recurrence and overcoming resistance to treatment is crucially needed for glioblastoma patients. Novel therapeutic approaches to control tumor recurrence by targeting crucial signaling molecules such TGF- $\beta$ (3), JAK-STAT (4-6) and WINT (7), adhesion molecules $\alpha \beta$ integrin (8), and pro-angiogenic factors VEGF and VEGFR (9), have been tried. However, a new breakthrough has yet to be found.

Immunotherapy is also recognized as a therapeutic tool against human GBM, and preliminary studies trying to suppress recurrence have been reported (10-13).

Ardon et al (13) reported that dendritic cell (DC)-based vaccines against GBM had moderate effects in terms of a patient's performance status (PS) or quality of life (QOL) in clinical phase I/II trial against advanced or newly diagnosed high-grade gliomas.

As to immunogenic antigens, several studies regarding glioma-specific cancer antigen including WT1, MAGE, gp100, HER2, which have been already used for clinical trial, were reported (14-16). WT-1 expression in glioblastomas was reported by Oji et al (14). Liu et al (16) reported that HER2, gp100 and MAGE-A1 mRNA expression was detected in 81.4, 46.5 and $39.5 \%$ of 43 GBM primary cell lines, and determined CTL epitope specific for each antigen. As to the CT antigen expression in gliomas, several studies have been reported (17-22). Debinski et al (17) showed the specific expression of 
Table I. Characteristics of the high-grade glioma patients.

\begin{tabular}{lc}
\hline Total no. enrolled & 17 \\
Age (years) & $55.3 \pm 16.6$ \\
Gender & Male (13), Female (4) \\
Pathological & GBM 13 (grade IV), AA 1 (grade III), \\
diagnosis & AO 3 (grade III)
\end{tabular}

GBM, glioblastoma multiforme; AO, anaplastic pligodendroglioma; AA, anaplastic astrocytoma.

IL-13RA2 gene, common CT antigen in high-grade gliomas. Recently, Syed et al (21) demonstrated that two CT antigens (MAGE-A1 and MAGE-A3) and gp100 may be candidate antigens in primary GBM cell lines using RT-PCR specific for $11 \mathrm{CT}$ antigens and 4 melanocyte-differentiation antigens, however, those antigen expression was low-to-variable level. Furthermore, Sahin et al (22) analyzed expression of 7 CT antigens in 88 brain tumor specimen including meningiomas, and showed that SCP-1 exhibited the highest expression rate. However, a comprehensive expression analysis of CT antigens in high-grade gliomas is very limited.

In the present study, a comprehensive expression analysis of $54 \mathrm{CT}$ antigens and 13 glioma-associated antigens were performed using a quantitative PCR in 17 high-grade gliomaderived primary cell lines, and the co-relation of antigen expression to overall survival and $\mathrm{O}^{6}$-methylguanine-DNAmethyltransferase (MGMT) expression was also investigated.

\section{Materials and methods}

Establishment of primary $G B$ cell lines from high-grade glioma patients. Glioma tumor samples were obtained from surgical resections by neurosurgeons in Shizuoka Cancer Center. The clinical research using tumor tissues from glioma patients was approved by the Institutional Review Board of Shizuoka Cancer Center, Shizuoka, Japan. All patients gave written informed consent. Seventeen high-grade glioma patients, who are sources of established primary glioma cell lines, are characterized in Table I.

Tumors were dissociated by teasing with forceps to make a single cell suspension, and plated in $25 \mathrm{~cm}^{2}$ culture flask in Dulbecco's modified Eagle's medium (DMEM) (Sigma, St. Louis, MO, USA) supplemented with $10 \%$ fetal bovine serum (FBS; Invitrogen), penicillin, streptomycin and gentamycin (Invitrogen) for serum-derived GB cell line. Glioblastoma cell lines (A172, T98G, LN-18, U87, U118, U138 and U373) was purchased from the American Type Culture Collection (ATCC; Manassas, VA, USA) and maintained in DMEM containing $10 \%$ FBS plus penicillin and streptomycin.

Antibodies and reagents. Antibodies against $6 \mathrm{CT}$ antigens (MAGE-A12, BAGE, DDX43, IL-13RA2, CTAGE1 and CASC5) were purchased from the following sources; Abnova for MAGE-A12, LSBio Inc. for BAGE, Proteintech for DDX43 and IL-13RA2, Santa Cruz Biotechnology for CTAGE1, Bethyl Laboratories Inc. for CASC5. Antibodies against 10 glioma-associated antigens (HER2, Ki-67,
Podplanin, gp100, GFAP, MGMT, Nestin, Olig2, S100 and CD133) were purchased from the following sources; Dako for HER2, Ki-67 and gp100, Santa Cruz Biotechology for Podplanin, AbD Serotec for GFAP, Abcam for MGMT and IBL for Nestin, Olig2 and CD133.

Quantitative polymerase chain reaction ( $q P C R)$ analysis. The real-time PCR analysis of CT antigen and glioma-associated genes using the 7500 Real-Time PCR System (Applied Biosystems, Foster City, CA, USA) was performed as previously described. Briefly, 54 CT antigen-specific PCR primers (from CT1.1 to CT50; shown in Table II) or 13 glioma-associated gene-specific PCR primers (HER2, Ki-67, Podplanin, gp100, GFAP, MGMT, Nestin, Olig2, S100, CD133, WT1, MART-1 and Tyrosinase) and TaqMan probes were purchased from Applied Biosystems. Total RNAs were extracted from each glioma cell line or primary cells derived from high-grade gliomas. Complementary DNA was synthesized from $100 \mathrm{ng}$ of the total RNA and quantitative PCRs were carried using a TaqMan RNA-to-CT 1-Step kit (Applied Biosystems). As a negative control for tumor RNA, total RNA from the normal fetal brain (platinum total RNA) was purchased from Takara Bio Inc. (Otsu, Shiga, Japan).

Immunohistochemistry (IHC). Two representative cases, GB-case no. 16 and 9-derived resected tumors were fixed with formalin solution and used for IHC analysis of CT antigens and glioma-associated antigens, respectively. Hematoxylineosin staining was performed according to the manufacturer's instructions. For immunostaining, antibodies against $6 \mathrm{CT}$ antigens (MAGE-A12, BAGE, DDX43, IL-13RA2, CTAGE1 and CASC5) and antibodies against 10 glioma-associated antigens (HER2, Ki-67, Podplanin, gp100, GFAP, MGMT, Nestin, Olig2, S100 and CD133) were used as primary antibody. Goat anti-mouse IgG antibody or goat anti-rabbit IgG was used as secondary antibody. Horseradish peroxidase (HRP) and hydrogen peroxide were utilized for making color according to the manufacturer's instructions (Vectastatin ABC kit; Vector Laboratories, Burlingame, CA, USA).

Statistical analysis. The overall survival analysis based on the REMBRANDT dataset regarding target antigen genes was examined by comparing differences in median survival time (MST) via the Kaplan-Meier method. A comparative analysis of survival times between groups was then performed using the log rank test. Values of $\mathrm{P}<0.05$ were considered statistically significant. For the correlation analysis to MGMT expression, the correlation coefficient, $r$, was calculated and statistical difference was analyzed using the Pearson's correlation test. Values of $\mathrm{P}<0.05$ were considered statistically significant.

\section{Results}

Establishment of high-grade glioma primary cell lines. Seventeen serum-derived primary cell lines were established. These cell lines consisted of 3 anaplastic oligodendrogliomas, 1 anaplastic astrocytoma and 13 GBMs. Histologically, 13 of the 17 cases were WHO grade IV. Morphological appearances seem to be mainly adherent, but different among 17 primary cell lines ranging from round to fibroblast-like shape. 
Table II. CT antigen PCR primer list.

\begin{tabular}{|c|c|c|c|c|c|}
\hline CT code & Gene family & CT code & Gene family & CT code & Gene family \\
\hline CT1.1 & MAGE-A1 & CT7.2 & MAGE-C3 & СТ29 & CASC5 \\
\hline CT1.2 & MAGE-A2 & СT8 & SYCP1 & СТ30 & TFDP3 \\
\hline CT1.3 & MAGE-A3 & СТ9 & BRDT & CT32 & LDHC \\
\hline CT1.4 & MAGE-A4 & CT10 & MAGE-C2 & СТ33 & MORC1 \\
\hline CT1.5 & MAGE-A5 & CT11.1 & SPANXA1 & СТ34 & DKKL1 \\
\hline CT1.6 & MAGE-A6 & CT12.1a & XAGE1 & СТ35 & SPO11 \\
\hline CT1.8 & MAGE-A8 & CT13 & DDX43 & СТ36 & CRISP2 \\
\hline CT1.9 & MAGE-A9 & CT14 & SAGE1 & СТ37 & FMR1NB \\
\hline CT1.10 & MAGE-A10 & CT15 & ADAM2 & CT38 & FTHL17 \\
\hline CT1.11 & MAGE-A11 & CT16.1 & PAGE5 & СТ39 & NXF2 \\
\hline CT1.12 & MAGE-A12 & CT17 & LIPI & CT41.1 & TDRD1 \\
\hline CT2.1 & BAGE & CT19 & IL-13RA2 & CT42 & TEX15 \\
\hline СТ3.1 & MAGE-B1 & CT21.1 & CTAGE1 & CT43 & FATE1 \\
\hline СТ3.2 & MAGE-B2 & СТ24.1 & CSAG1 & CT44 & TPTE \\
\hline CT3.4 & MAGE-B6 & CT25.1a & DSCR8 & CT45 & CT45A1-6 \\
\hline CT4.1 & GAGE1 & CT26 & DDX53 & CT47.11 & CT47A11 \\
\hline CT5.1 & SSX1 & CT27 & CTCFL & CT48 & SLC06A1 \\
\hline СТ6.1 & CTAG1B & CT28 & LUZP4 & СТ50 & LEMD1 \\
\hline
\end{tabular}

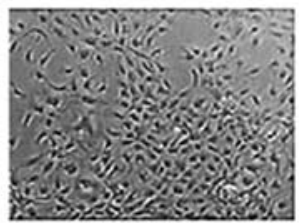

GB.SCC003T

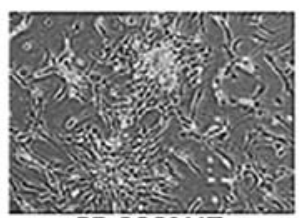

GB.SCC011T

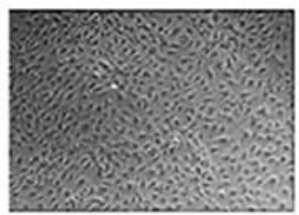

GB.SCC022T

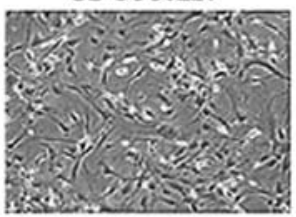

GB.SCC029T

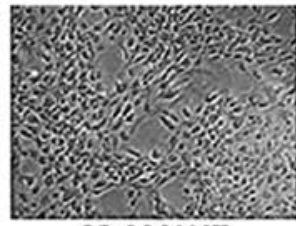

GB.SCC006T

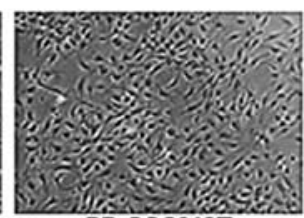

GB.SCC013T

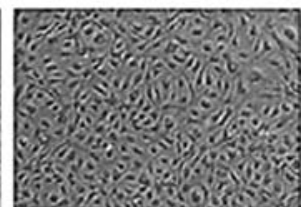

GB.SCC024T

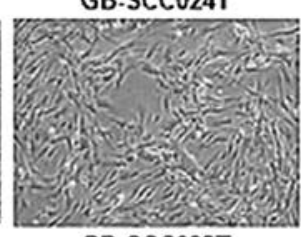

GB.SCC032T

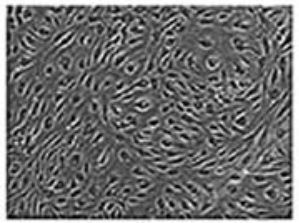

GB.SCC007T

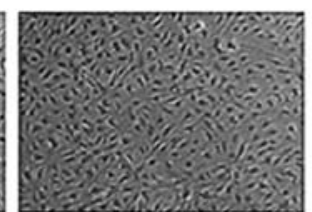

GB.SCC017T

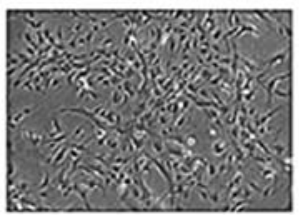

GB.SCC026T

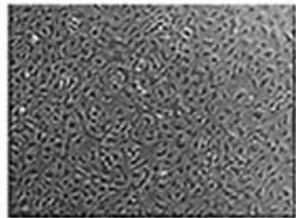

GB.SCC008T

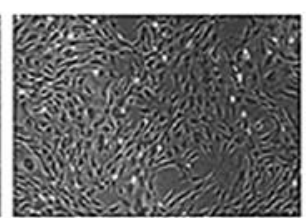

GB.SCC019T

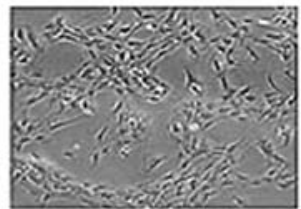

GB.SCC027T

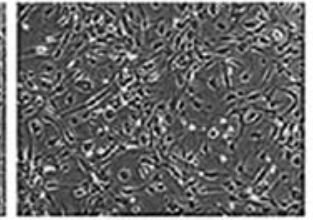

GB.SCC010T

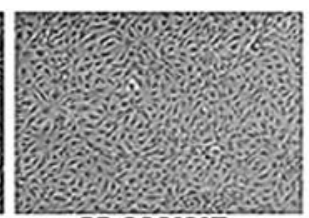

GB.SCCO20T

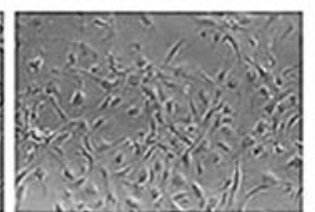

GB.SCC028T

Figure 1. Morphological appearance of the 17 established primary high-grade glioma cell lines. These cell lines consisted of 3 anaplastic oligodendrogliomas, 1 anaplastic astrocytoma and 13 GBMs. Morphological appearance is mainly adherent, but different among the 17 primary cell lines varying from round to fibroblast-like shape. Original magnification, x100.

Heterogeneous cell populations were seen, where adherent and floating cells were mixed (Fig. 1).

Quantitative PCR analysis of CT antigen or glioma-associated gene $m R N A$ in glioma primary cell lines. A quantitative
PCR using 54 cancer-testis (CT) antigen-specific primers showed that $36 \mathrm{CT}$ antigens were positive in at least 1 of 17 serum-derived cell lines, and 17 antigens were positive in $>50 \%$ cell lines (Table III). Impressively, 6 genes (BAGE, MAGE-A12, CSAC5, CTAGE1, DDX43 and IL-13RA2) 
Table III. CT antigen expression in glioma cell lines and primary cells from high-grade glioma patients.

\begin{tabular}{|c|c|c|c|c|c|c|c|c|}
\hline Antigen & LN-18 & U87 & A172 & T98 & U118 & U138 & U373 & GB primary \\
\hline MAGEA12 & + & + & + & + & + & + & + & $17(100 \%)$ \\
\hline CSAC5 & + & + & + & + & + & + & + & $17(100 \%)$ \\
\hline CTAGE1 & + & + & + & + & + & + & + & $17(100 \%)$ \\
\hline DDX43 & + & + & + & - & - & - & + & $17(100 \%)$ \\
\hline IL13RA2 & + & + & + & + & + & + & + & $17(100 \%)$ \\
\hline BAGE & + & + & + & + & + & + & + & $17(100 \%)$ \\
\hline CSAG1 & + & + & + & + & + & + & + & $16(94 \%)$ \\
\hline LDHC & + & + & + & + & + & + & + & $16(94 \%)$ \\
\hline TDRD1 & + & - & + & - & - & - & - & $16(94 \%)$ \\
\hline MAGEB6 & + & + & + & + & + & + & + & $16(94 \%)$ \\
\hline TEX15 & + & + & - & + & + & - & + & $15(88 \%)$ \\
\hline TFDP3 & + & + & + & + & + & + & + & $15(88 \%)$ \\
\hline SPANXA1 & + & + & + & + & + & + & + & $14(82 \%)$ \\
\hline CTCFL & + & + & + & - & - & - & + & $13(76 \%)$ \\
\hline MAGEA11 & + & + & + & - & + & - & - & $13(76 \%)$ \\
\hline MORC1 & + & + & + & + & + & + & + & $12(70 \%)$ \\
\hline FMR1NB & + & - & + & + & + & + & + & $11(64 \%)$ \\
\hline DDX53 & + & + & + & + & + & + & + & $8(47 \%)$ \\
\hline CTAG1B & + & - & - & - & - & - & - & $5(29 \%)$ \\
\hline FTHL17 & + & + & + & - & - & - & + & $5(29 \%)$ \\
\hline MAGEC2 & + & + & - & + & - & - & - & $4(23 \%)$ \\
\hline FATE1 & + & + & - & + & + & + & - & $4(23 \%)$ \\
\hline SAGE1 & + & + & - & - & - & - & - & $3(18 \%)$ \\
\hline XAGE1A-E & + & + & + & + & + & + & - & $3(18 \%)$ \\
\hline CT47A11 & + & + & - & + & + & + & - & $3(18 \%)$ \\
\hline SLC06A1 & + & - & - & - & - & - & - & $3(18 \%)$ \\
\hline CRISP2 & + & - & + & - & - & + & - & $2(11 \%)$ \\
\hline LEMD & - & - & + & + & - & - & - & $2(11 \%)$ \\
\hline LUZP4 & + & + & - & - & - & + & - & $2(11 \%)$ \\
\hline CT45A1-6 & + & + & - & - & - & - & - & $2(11 \%)$ \\
\hline NXF & + & + & + & - & + & - & - & $1(5.8 \%)$ \\
\hline BRDT & - & - & - & - & + & + & + & $1(5.8 \%)$ \\
\hline MAGEA3 & + & + & + & + & + & + & + & $1(5.8 \%)$ \\
\hline SSX & + & + & + & + & + & + & - & $1(5.8 \%)$ \\
\hline ADAM2 & + & - & - & - & - & - & - & $1(5.8 \%)$ \\
\hline SPO11 & - & - & - & + & - & - & - & $1(5.8 \%)$ \\
\hline
\end{tabular}

were detected in all the cell lines. On the other hand, the expression of other 13 glioma-associated antigens than CT genes were also investigated, and 10 genes were detected in $>70 \%$ of the cell lines (Table IV). Glioma-specific antigens CD133, S100, Olig2, Podplanin and Nestin were detected in most primary cells as well as commercially available cell lines. Notably, gp100 and HER2, common cancer antigens, were also positively expressed in all cell lines as well as the regular ones. MGMT was positive in all cell lines except one.

Immunohistochemistry. The expression of CT antigen and glioma-associated antigen genes with a high frequency were verified in IHC analysis using GB-case 16-derived resected tumor specimen. In $6 \mathrm{CT}$ antigens (MAGE-A12, BAGE, DDX43, IL-13RA2, CTAGE1 and CASC5), all antigen proteins other than CTAGE1 were positively expressed in the tumor from patient (Fig. 2). On the other hand, 10 gliomaassociated antigens (HER2, Ki-67, Podplanin, gp100, GFAP, MGMT, Nestin, Olig2, S100 and CD133) staining showed that all antigen proteins besides MGMT, CD133, HER2 and gp100 were detected in the tumor from GB-case 9 (Fig. 3).

CT antigen gene expression compared with normal brain tissue. Gene expression levels of $6 \mathrm{CT}$ antigens in primary high-grade glioma cell lines were significantly upregulated in CASC5 and CTAGE1 compared with normal brain (Fig. 4). In particular, CASC5 expression level was higher almost 10-fold 
Table IV. Glioma-associated gene expression in glioma cell lines and primary cells from high-grade glioma patients.

\begin{tabular}{lcccccccc}
\hline Antigen & LN-18 & U87 & A172 & T98 & U118 & U138 & U373 & GB primary \\
\hline HER2 & + & + & + & + & + & + & + & $17(100 \%)$ \\
Ki-67 & + & + & + & + & + & + & + & $17(100 \%)$ \\
Podoplanin & + & + & - & + & + & + & + & $17(100 \%)$ \\
gp100 & + & + & + & + & + & + & + & $17(100 \%)$ \\
MGMT & + & - & - & + & + & + & + & $16(94 \%)$ \\
NES & + & + & + & + & + & + & + & $16(94 \%)$ \\
GFAP & + & + & + & - & + & - & + & $15(88 \%)$ \\
OLIG2 & + & + & + & + & + & + & - & $15(88 \%)$ \\
S100 & + & - & + & + & + & + & + & $14(82 \%)$ \\
CD133 & + & + & + & + & + & + & + & $12(70 \%)$ \\
WT-1 & + & + & + & + & - & - & + & $8(47 \%)$ \\
MelanA & - & - & - & - & - & - & - & $2(11 \%)$ \\
Tyrosinase & - & + & - & - & + & - & - & $1(5.8 \%)$ \\
\hline
\end{tabular}

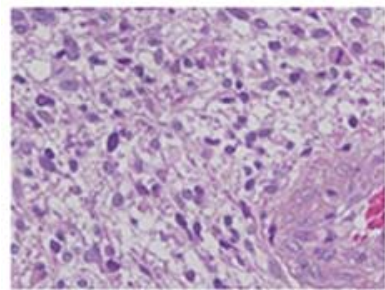

$\mathrm{HE}$

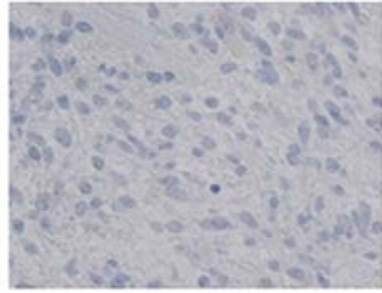

Control

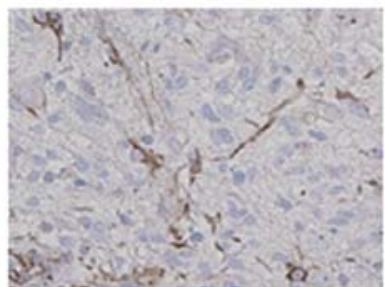

BAGE

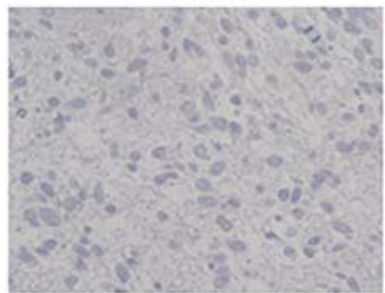

CTAGE1

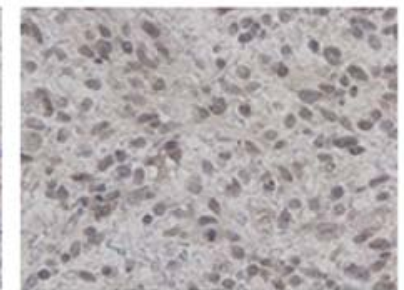

MAGE-A12

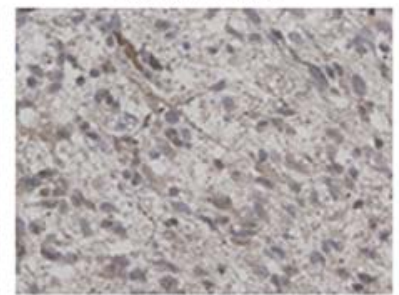

DDX43

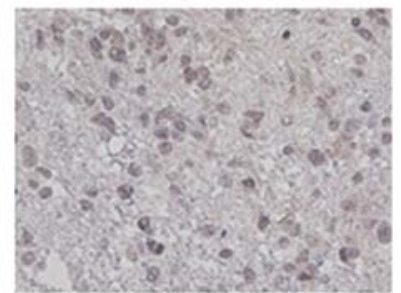

CASC5

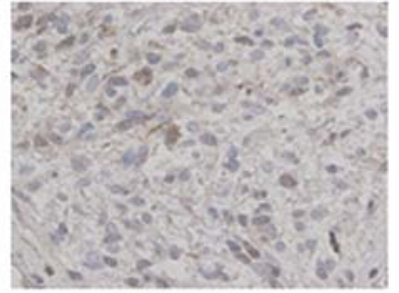

IL13RA2

Figure 2. CT antigen protein expression in GBM tumor tissue (GB-no. 16). Primary antibodies against 6 CT antigens (MAGE-A12, BAGE, DDX43, IL-13RA2, CTAGE1 and CASC5) were used for immunostaining. Expression of all the antigens, but CTAGE1, was positive. H\&E; hematoxylin-eosin, magnification, $\mathrm{x} 400$.

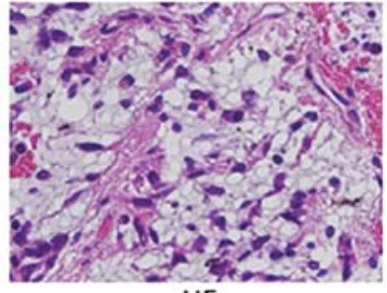

$\mathrm{HE}$
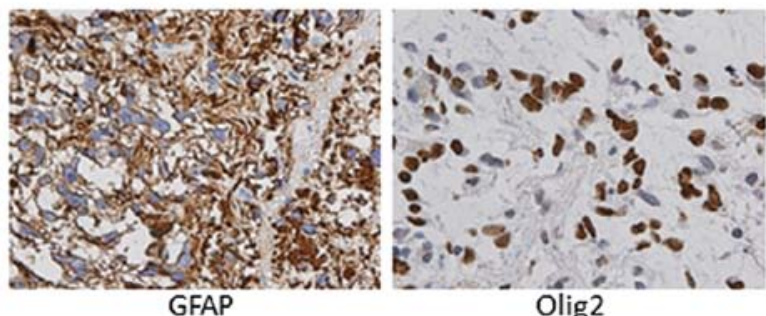

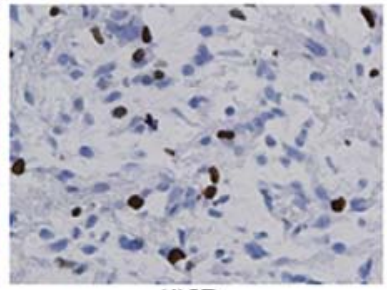

Ki67

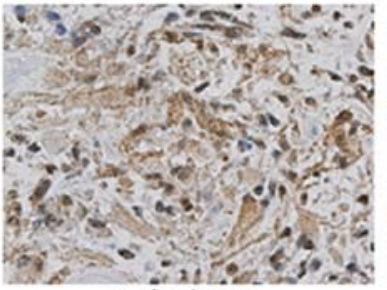

Podoplanin

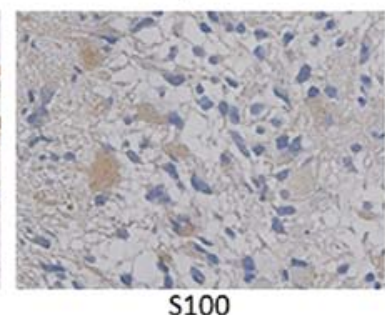

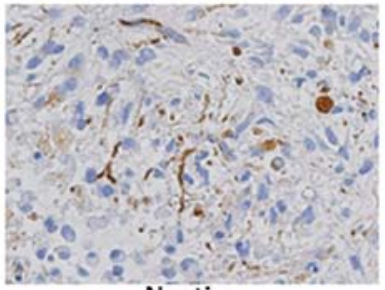

Nestin

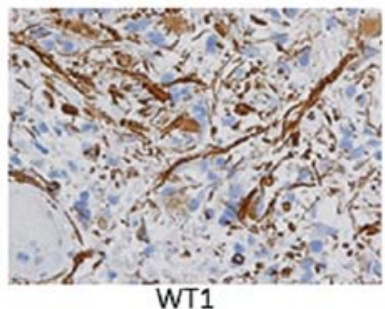

Figure 3. Glioma-associated antigen protein expression in GBM tumor tissue (GB-no. 9). Primary antibodies against 10 glioma-associated antigens (HER2, Ki-67, Podplanin, gp100, GFAP, MGMT, Nestin, Olig2, S100 and CD133) were used for immunostaining. Expression of MGMT, gp100 and CD133 was negative, and for all other antigens positive. H\&E; hematoxylin-eosin; magnification, $x 400$. 


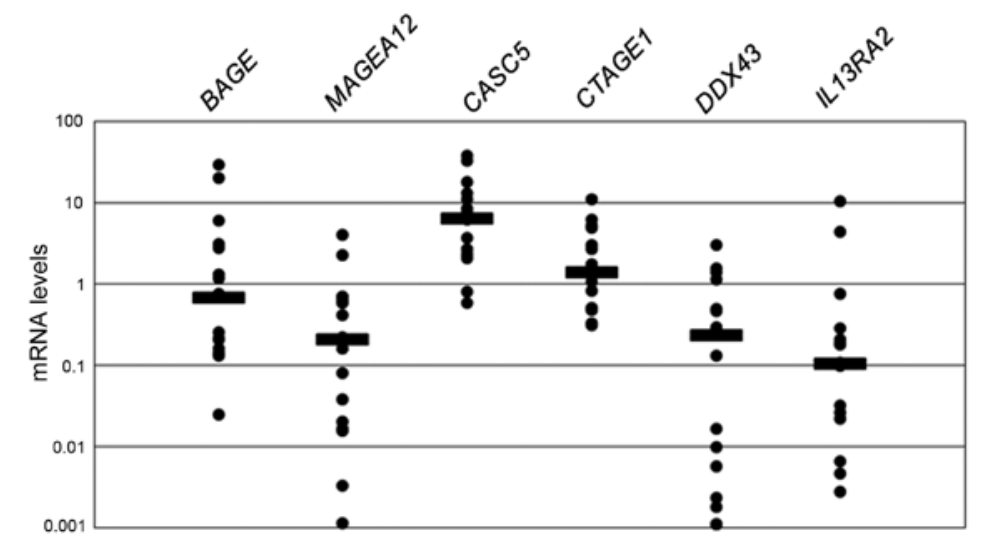

Figure 4. Expression of six CT antigens (MAGE-A12, BAGE, DDX43, IL-13RA2, CTAGE1 and CASC5) in 17 high-grade glioma cell lines compared with normal brain tissue. Quantitative PCR analyses of 6 CT antigen genes were performed and normalized with GAPDH mRNA level. Each CT antigen expression level in normal brain tissue was rated as 1 .

Table V. Prognostic factor candidates.

\begin{tabular}{lccc}
\hline $\begin{array}{l}\text { Gene } \\
\text { family }\end{array}$ & $\begin{array}{c}\text { Positive } \\
\text { rate }(\%)\end{array}$ & $\begin{array}{c}\text { Gene } \\
\text { expression }\end{array}$ & P-value \\
\hline IL-13RA2 & $17 / 17(100)$ & Overexpressed & $2.08 \times 10^{-4 a}$ \\
HER2 & $17 / 17(100)$ & Overexpressed & $8.53 \times 10^{-7 a}$ \\
Ki-67 & $17 / 17(100)$ & Overexpressed & $2.57 \times 10^{-3 a}$ \\
Podplanin & $17 / 17(100)$ & Overexpressed & $7.02 \times 10^{-14 a}$ \\
Olig2 & $15 / 17(88)$ & Overexpressed & $1.75 \times 10^{-10 a}$ \\
CD133 & $12 / 17(70)$ & Overexpressed & $8.1 \times 10^{-8 a}$ \\
\hline
\end{tabular}

Kaplan-Mayer plot was performed using REMBRANDT analysis on NCBI data base. Survival time between overexpressed ( $>2.0$ fold) and underexpressed $(<2.0$-fold) group was evaluated by Wilcoxon $\log$ rank test. ${ }^{\mathrm{a}} \mathrm{P}<0.01$.

than normal brain. Unexpectedly, IL-13RA2 mean expression level was lower than normal level.

Correlation of CT antigen expression to overall survival and MGMT expression. The correlation of antigen expression level to overall survival time was analyzed using REMBRANDT database of the National Cancer Institute in the 17 CT antigens and 10 glioma-associated genes which were highly expressed in glioma primary cell lines. The expressions of IL-13RA2, HER2, Ki-67, podplanin, Olig2 and CD133 were inversely correlated to overall survival time in high-grade glioma patients given a standard chemo-radiation after surgery $(\mathrm{P}<0.01$; Table V). Another correlation analysis of CT antigen and glioma-associated gene to MGMT expression demonstrated that 4 genes including DDX43, TDRD1, HER 2 and gp100 had significant positive relationship to MGMT expression. Particularly, HER 2 and gp100 genes showed very high coefficient $(r=0.751$ and 0.894 , respectively; Table VI).

\section{Discussion}

Glioblastoma multiforme (GBM) is one of the most malignant and aggressive tumors, and even with an intensive treatment
Table VI. Relevant factors to MGMT expression.

\begin{tabular}{lccr}
\hline $\begin{array}{l}\text { Gene } \\
\text { family }\end{array}$ & $\begin{array}{c}\text { Positive } \\
\text { rate (\%) }\end{array}$ & $\begin{array}{c}\text { Correlation } \\
\text { coefficient (r) }\end{array}$ & \multicolumn{1}{c}{ P-value } \\
\hline DDX43 & $17 / 17(100)$ & 0.540 & $0.0292^{\mathrm{a}}$ \\
TDRD1 & $16 / 17(94.1)$ & 0.675 & $0.0031^{\mathrm{b}}$ \\
HER2 & $17 / 17(100)$ & 0.751 & $0.0004^{\mathrm{b}}$ \\
gp100 & $17 / 17(100)$ & 0.894 & $<0.0001^{\mathrm{b}}$ \\
\hline
\end{tabular}

The correlation coefficient, $r$, was calculated and statistical difference was analyzed using the Pearson's correlation test. ${ }^{a} \mathrm{P}<0.05$, ${ }^{\mathrm{b}} \mathrm{P}<0.01$

including surgery, radiation and chemotherapy, the median survival time is about 1 year and few cases can survive more than 2 fears $(1,2)$. Therefore, novel therapeutic approach to control the recurrence and overcome the resistance to treatment is crucially needed in glioblastoma patients.

A prerequisite for the successful immunotherapy is the identification of suitable tumor antigens specifically expressed in cancer tissues and its clinical application as an advanced translational research. Renkvist et al (23) classified human tumor antigens recognized by $\mathrm{T}$ cells into 6 categories; i) HLA class I-restricted cancer/testis antigens, ii) differentiation antigens, iii) widely expressed antigens, iv) tumor-specific antigens, v) HLA class II-restricted antigens and (vi) fusion proteins. Particularly, CT antigen and melanoma-associated differentiation antigen have been focused on, and several peptides including MAGE family, NY-ESO, IL-13RA2, MART-1, gp100 and tyrosinase were effectively used in clinical vaccinations for advanced melanoma and glioma patients (24-28).

To develop novel glioma-specific antigens, in the present study, we performed a comprehensive expression analysis of CT antigen and glioma-associated genes in patients-derived primary glioma cell lines using a quantitative PCR. A quantitative PCR using 54 cancer-testis (CT) antigen-specific primers showed that $36 \mathrm{CT}$ antigens were positive in at least 1 of 17 serum-derived cell lines, and 17 antigens were positive 
in $>50 \%$ cell lines. Particularly, 6 genes (BAGE, MAGE-A12, CSAC5, CTAGE1, DDX43 and IL-13RA2) were detected in all cell lines. Sahin et al (22) reported the frequency of selected $7 \mathrm{CT}$ antigen gene expression in 88 human brain tumor specimen using RT-PCR. The highest frequency was $40 \%$ in SCP-1 gene expression, and 23 astrocytomas expressed at least one CT gene. Another RT-PCR study by Syed et al (21) using 45 glioma-frozen tissues revealed that the highest $\mathrm{CT}$ antigen expression rates were MAGEA-3 (22\%) and MAGEA-1 (16\%) in $11 \mathrm{CT}$ genes.

Our highest expression rate was $100 \%$ in 6 CT genes, and all primary cell lines showed expression in at least 14 $\mathrm{CT}$ antigen genes, thus showing significantly better results compared to the previous study. Possible reasons why our expression frequency was higher is speculated as; i) higher purity of tumor cells because newly established serum-derived primary cell cultures were used for analysis, ii) the number of CT genes analyzed was higher, iii) validated quantitative PCR primer. On the other hand, the expression of other 13 glioma-associated antigens than CT genes were also investigated, and 10 genes were detected in more than $70 \%$ of the cell lines. Specifically, glioma-assocated genes such as HER2, podoplanin and gp100 were positive in all the cell lines.

HER2 and gp100 as melanocyte-differentiation antigen (MDA) were reported to be highly expressed in malignant melanoma and glioma tissues as well as WT1. Interestingly, because both melanocytes and glial cells are derived from the neuroectoderm, it is not surprising that they express common MDA and CT antigen genes (29).

In the present study, correlation analysis of $\mathrm{CT}$ antigen and glioma-associated genes to overall survival time and MGMT expression was performed. IL-13RA2 and HER2 genes were demonstrated to be prognostic factor candidates for a poor survival (Table V), which may suggest that prognostic analysis can contribute to the understanding the novel function of $\mathrm{CT}$ antigen genes. Both target genes are well known to be expressed in GBM tumors, therefore specific clinical immunotherapy against these molecules has been performed in a small size of trail design. Specifically, obvious evidence that the downregulation of IL-13RA2 and HER2 antigen expression by therapeutic agents may contribute to a good prognosis has yet to be obtained. Furthermore, few studies regarding the correlation between CT antigens and survival time have been reported to date. That is why our observations should be stressed in terms of novelty.

Additionally, we analyzed the correlation of CT antigen genes to MGMT expression. Interestingly, 4 antigen gene candidates positively correlated to MGMT expression, were identified. In particular, HER2 and gp100 showed a high coefficient ( $\mathrm{r}=0.751$ and 0.894 , respectively). MGMT expression is considered to be regulated by DNA methylation mechanism, and crucial in terms of determining the chemosensitivity of high-grade gliomas to temozolomide (30). It is well-known that high expression of MGMT is closely associated with poor prognosis in high-grade gliomas, and DNA-methylation of MGMT can be a possible approach to MGMT reduction $(31,32)$.

If some CT antigens are linked to MGMT gene in DNA-demethylation to promoter area, epigenetic approach to DNA methylation of CT genes could be a novel therapy, resulting in a therapeutic effect on high-grade gliomas. However, there is no evidence for correlation of both gene promoters, and clarification of the question will need more investigation. Natsume et al (33) reported that the DNA-demethylating agent 5-aza-2'-deoxycytidine (5-aza-CdR) remarkably reactivated NY-ESO-1 gene expression and demonstrated that 5-aza-CdRinduced expression of epigenetically silenced $\mathrm{CT}$ antigen gene might be a new strategy for immunotherapy against high-grade glioma.

Little is known about the precise function of CT antigen genes. Notably, Low et al (34) reported a novel approach to evaluating the function of CT gene using knockdown technology against neural stem cell-derived CT antigen, and showed some $\mathrm{CT}$ antigen genes were associated with the stem cell differentiation.

Finally, in the present study, we analyzed expression of $54 \mathrm{CT}$ antigens using a quantitative PCR. In near future, CT antigen repertoire will be expanded up to 100, and more efficient biomarkers and prognostic markers specific for high-grade glioma should be collected, where effective target antigens can be determined for developing novel immunotherapy and antibody medicines.

\section{Acknowledgements}

The present study was supported by a grant from the Regional Innovation Strategy Support Program of the Ministry of Education, Culture, Sports, Science and Technology, Japan.

\section{References}

1. Stupp R, Mason WP, van den Bent MJ, et al: Radiotherapy plus concomitant and adjuvant temozolomide for glioblastoma. $\mathrm{N}$ Engl J Med 352: 987-996, 2005.

2. Mirimanoff RO, Gorlia T, Mason W, et al: Radiotherapy and temozolomide for newly diagnosed glioblastoma: recursive partitioning analysis of the EORTC 26981/22981-NCIC CE3 phase III randomized trial. J Clin Oncol 24: 2563-2569, 2006.

3. Ikushima H, Todo T, Ino Y, Takahashi M, Miyazawa K and Miyazono K: Autocrine TGF- $\beta$ signaling maintains tumorigenicity of glioma-initiating cells through Sry-related HMG-box factors. Cell Stem Cell 5: 504-514, 2009.

4. McFarland BC, Ma JY, Langford CP, Gillespie GY, Yu H, Zheng Y, Nozell SE, Huszar D and Benveniste EN: Therapeutic potential of AZD1480 for the treatment of human glioblastoma. Mol Cancer Ther 10: 2384-2393, 2011.

5. Sai K, Wang S, Balasubramaniyan V, et al: Induction of cell-cycle arrest and apoptosis in glioblastoma stem-like cells by WP1193, a novel small molecule inhibitor of the JAK2/STAT3 pathway. J Neurooncol 107: 487-501, 2012.

6. Ashizawa T, Miyata H, Ishii H, et al: Antitumor activity of a novel small molecules STAT3 inhibitor against a human lymphoma cell line with high STAT3 activation. Int J Oncol 38: 1245-1252, 2011.

7. Zhou Y, Liu F, Xu Q and Wang X: Analysis of the expression profile of Dickkopf-1 gene in human glioma and the association with tumor malignancy. J Exp Clin Cancer Res 29: $138,2010$.

8. Reardon DA, Fink KL, Mikkelsen T, et al: Randomized phase II study of cilemgitide, an integrin-targeting arginine-glycineaspartic acid peptide, in recurrent glioblastoma multiforme. J Clin Oncol 26: 5610-5617, 2008.

9. Jo J, Schiff D and Purow B: Angiogenic inhibition in high-grade gliomas: past, present and future. Expert Rev Neurother 12: 733-747, 2012.

10. Chang CN, Huang YC, Yang DM, Kikuta K, Wei KJ, Kubota T and Yang WK: A phase I/II clinical trial investigating the adverse and therapeutic effects of a postoperative autologous dendritic cell tumor vaccine in patients with malignant glioma. J Clin Neurosci 18: 1048-1054, 2011. 
11. Luptrawan A, Liu G and Yu JS: Dendritic cell immunotherapy for malignant gliomas. Rev Recent Clin Trials 3: 10-21, 2008.

12. Ardon H, Van Gool SW, Verschuere T, et al: Intergation of autologous dendritic cell-based immunotherapy in the standard of care treatment for patients with newly diagnosed glioblastoma: results of the HGG-2006 phase I/II trial. Cancer Immunol Immunother 61: 2033-2044, 2012.

13. Ardon H, Van Gool S, Lopes IS, et al: Integration of autologous dendritic cell-based immunotherapy in the primary treatment for patients with newly diagnosed glioblastoma multiforme: a pilot study. J Neurooncol 99: 261-272, 2010.

14. Oji Y, Suzuki T, Nakano Y, et al: Overexpression of the Wilms tumor gene WT1 in primary astrocytic tumors. Cancer Sci 95: 822-827, 2004.

15. Bodey B, Siegel SE and Kaiser HE: MAGE-1, a cancer/testisantigen, expression in childhood astrocytomas as an indicator of tumor progression. In vivo 16: 583-588, 2002.

16. Liu G, Ying H, Zeng G, Wheeler CJ, Black KL and Yu JS: HER-2, gp100, and MAGE-1 are expressed in human glioblastoma and recognized by cytotoxic T cells. Cancer Res 64: 4980-4986, 2004.

17. Debinski W and Gibo DM: Molecular expression analysis of restrictive receptor for interleukin 13, a brain tumor-associated cancer/testis antigen. Mol Med 6: 440-449, 2000.

18. Schmits R, Cochlovius B, Treitz G, et al: Analysis of the antibody repertoire of astrocytoma patients against antigens expressed by gliomas. Int J Cancer 98: 73-77, 2002.

19. Yawata T, Nakai E, Park KC, et al: Enhanced expression of cancer testis antigen genes in glioma stem cells. Mol Carcinog 49: 532-544, 2010.

20. Oba-Shinjo SM, Caballero OL, Jungbluth AA, Rosemberg S, Old LJ, Simpson AJ and Marie SK: Cancer-testis (CT) antigen expression in medulloblastoma. Cancer Immun 8: 7, 2008.

21. Syed ON, Mandigo CE, Killory BD, Canoll P and Bruce JN: Cancer-testis and melanocyte-differentiation antigen expression in malignant glioma and meningioma. J Clin Neurosci 19: 1016-1021, 2012.

22. Sahin U, Koslowski M, Tureci O, et al: Expression of cancer testis genes in human brain tumors. Clin Cancer Res 6: 3916-3922, 2000.

23. Renkvist N, Castelli C, Robbins PF and Parmiani G: A listing of human tumor antigens recognized by $\mathrm{T}$ cells. Cancer Immunol Immunother 50: 3-15, 2001.
24. Nestle FO, Alijagic S, Gilliet M, et al: Vaccination of melanoma patients with peptide- or tumor lysate-pulsed dendritic cells. Nat Med 4: 328-332, 1998

25. Akiyama Y, Tanosaki R, Inoue N, et al: Clinical response in Japanese metastatic melanoma patients treated with peptide cocktail-pulsed dendritic cells. J Transl Med 3: 4, 2005.

26. Tyagi $P$ and Mirakhur B: MAGRIT: the largest-ever phase III lung cancer trial aims to establish a novel tumor-specific approach to therapy. Clin Lung Cancer 10: 371-374, 2009.

27. Aoki M, Ueda S, Nishikawa $\mathrm{H}$, et al: Antibody responses against NY-ESO-1 and HER2 antigens in patients vaccinated with combinations of cholesteryl pullulan (CHP)-NY-ESO-1 and CHP-HER2 with OK-432. Vaccine 27: 6854-6861, 2009.

28. Okada H, Kalinski P, Ueda R, et al: Induction of CD8 ${ }^{+} \mathrm{T}$-cell responses against novel glioma-associated antigen peptides and clinical activity by vaccinations with $\alpha$-type 1 polarized dendritic cells and polyinosinic-polycytidylic acid stabilized by lysine and carboxymethylcellulose in patients with recurrent malignant glioma. J Clin Oncol 29: 330-336, 2011.

29. Chi DD, Merchant RE, Rand R, et al: Molecular detection of tumor-associated antigens shared by human cutaneous melanomas and gliomas. Am J Pathol 150: 2143-2152, 1997.

30. Hegi ME, Diserens AC, Gorlia T, et al: MGMT gene silencing and benefit from temozolomide in glioblastoma. N Engl J Med 352: 997-1003, 2005.

31. Hegi ME, Liu L, Herman LG, et al: Correlation of $\mathrm{O}^{6}$-methylguanine methyltransferase (MGMT) promoter methylation with clinical outcomes in glioblastoma and clinical strategies to modulate MGMT activity. J Clin Oncol 26: 4189-4199, 2008.

32. Quinn JA, Jiang SX, Reardon DA, et al: Phase II trial of temozolomide plus $\mathrm{O}^{6}$-benzylguanine in adults with recurrent, temozolomide-resistant maliganant glioma. J Clin Oncol 27: 1262-1267, 2009

33. Natsume A, Wakabayashi T, Tsujimura K, et al: The DNA demethylating agent 5-aza-2'-deoxycytidine activates NY-ESO-1 antigenicity in orthotopic human glioma. Int $\mathrm{J}$ Cancer 122: 2542-2553, 2008.

34. Low J, Dowless M, Shiyanova T, et al: Knockdown of cancer testis antigens modulates neural stem cell marker expression in glioblastoma tumor stem cells. J Biomol Screen 15: 830-839, 2010 . 